\title{
Overcoming Diffusion Barriers of Digital Health Innovations: Conception of an Assessment Method
}

\author{
Richard Hobeck \\ Technische Universität Berlin \\ richard.hobeck@tu-berlin.de
}

\author{
Hannes Schlieter \\ Technische Universität Dresden \\ hannes.schlieter@tu-dresden.de
}

\author{
Tim Scheplitz \\ Technische Universität Dresden \\ tim.scheplitz@tu-dresden.de
}

\begin{abstract}
Digital health innovations (DHIs) contribute to improving the health sector by revitalizing availability and continuity of care as well as mitigating rising costs. DHIs getting increasing support from health insurance companies and governmental institutions, but still struggle on their way to standard care in national healthcare systems. One of the central challenges is the multitude of diffusion barriers, which are either little known or difficult to handle in complexity and therefore pose a high risk for the translation into the healthcare practice. This paper steps into this discourse with a design-oriented research approach. A systematic literature review identified DHI barriers that are further evolved to a concept for assessing barrier resilience. On that basis, a framework to systematically administer diffusion barriers to DHI in Germany was developed. Innovators may use the proposed framework to assess the likelihood of a successful implementation and to ensure smooth scaling up process of their DHI.
\end{abstract}

\section{Introduction}

Digital technology and the United Nations Sustainable Development Goals (SDGs) [1] are interwoven on a variety of levels. One example of this interplay is the third objective of the SDGs aiming at health-related action areas in which digital technology, in form of digital health, represents a significant element to achieve the formulated sustainability goals [2], [3]. Restraining this connection, however, digital healthcare solutions often face the scaling-up problem - a phenomenon that describes how digital health innovations (DHIs) are retained from finding their way into standard care. Solutions that have a demonstrable effect on care can frequently not unfold their benefits for the general public. Recently, regulatory measures such as the German Digital Healthcare Act show that political decisions support the digital transformation of the health sector and that digital health applications are acknowledged in care alongside medication [4]. Nevertheless, the basic problem remains: the complex and regulated healthcare environment imposes a large number of barriers to health innovations that are little known, especially to smaller innovators, and thus present a high risk to the successful development and exploitation of DHIs [5]. Small and medium-sized enterprises often struggle to identify such barriers and miss out on taking appropriate mitigation measures early on to ensure proper development and marketing of their solutions [6].

Therefore, we present an evaluation approach to support the early analysis and identification of possible deficits in targeting propagation barriers. Thus, we help to increase the accessibility of digital health applications and thereby the availability of healthcare for communities. Evaluation is used when informed decisions are to be made. The evaluation approach in this paper utilizes theoretical knowledge on propagation barriers of health innovations. Practitioners benefit from getting a concise outline of propagation-related strengths and weaknesses of their DHI in key figures. This can serve as a useful tool to develop sustainable digital health solutions that keep up with high expectations posed upon them and at the same time help researchers to further investigate the phenomenon of the scaling-up problem [7]. The design objective of this article is therefore linked to the question:

What does an approach to assess the barrier resilience of Digital Health Innovations look like?

A DHI in the context of this paper is the use of information and communication technology to deliver health or health-related services [3]. Nested in diffusion theory [8] and scaling-up of innovations [9], this paper builds on a body of preliminary work covering two domains: (1) diffusion barriers of DHIs and methodologies to reveal them as in analysing the lessons learned of DHI projects [10] and providing generic classifying taxonomies of DHI diffusion barriers [11]; and (2) a number of evaluation frameworks of DHIs. 
This paper complements recent initiatives to develop DHI evaluation frameworks [12], [13] by adding a categorization of existing evaluation approaches for health innovations and deriving an assessment framework from propagation barriers in healthcare. We add to the existing literature by newly accumulating knowledge from the two domains and providing an artifact as an applicable implementation of theory as design science [14]. The framework is exemplarily made-to-measure the German healthcare environment in order to show the applicability and utility of the approach in a concrete healthcare market. Generally, Germany can also serve as a representative example among OECD states with Social Health Insurance, i.e., societal actors decide on regulations and financing while services are largely provided by private for-profit actors [15]. The final assessment, however, needs to be tailored to the specific national context.

Starting with researching propagation barriers focusing on DHIs in Germany, the identified corpus of contributions centered around these barriers is examined. Next, we present our methodology (Section 2) and provide an overview of existing evaluation approaches (Section 3). Based on that, an assessment approach tailored to the German healthcare environment is designed (Section 4). The resulting framework is tested using an exemplary project in the German healthcare market with a phenotypical character for DHIs (Section 5). The paper closes with a discussion of the results (Section 6) and concluding remarks as well as an outlook on further research (Section 7).

\section{Methodology}

The methodological approach is based on the Design Science Research (DSR) principles [16] and aligned with a DSR process (DSRP) model [14]. The relevance and problem identification (DSRP Phase 1) as well as the design objective (DSRP Phase 2) was elaborated in Section 1. The artifact is constructed in multiple steps (DSRP Phase 3) as follows: In order to comply with DSR-guidelines and to ground the research on rigorous practices, a systematic literature search was carried out to accumulate literature broaching the issue of diffusion barriers in healthcare [17]. Based on this, the research results were analyzed using inductive category formation by Mayring to identify propagation barriers of DHIs in general and specific barriers in Germany [18]. Next, existing approaches to evaluating digital health solutions were identified in a second literature search. To map the ground covered by preliminary research, a taxonomy was created helping to categorize existing DHI assessment approaches [19]. The categorization revealed an existing artifact that is iterated to fit the needs of an evaluation tool synthesized for the German environment [20]. Finally, this paper's framework is applied (DSRP Phase 4) and evaluated (DSRP Phase 5) using a practical example.

Identification of propagation barriers. In order to identify existing literature on propagation barriers in the German healthcare system, scientific databases were searched for relevant contributions on August 4, 2018 [17], [21]. The search term consisted of three elements to which synonyms in German and English were added: (1) barriers (obstacles, hurdles, resistance, etc.), (2) propagation mechanisms (scaling-up, translation, diffusion, etc.) and (3) digital health innovations (eHealth, telemedicine, telehealth, etc.). The search queries were applied to the databases EBSCOhost, ScienceDirect, IEEE Xplore, SpringerLink, PubMed and AIS Electronic Library (+368). Duplicates, nonEnglish and non-German contributions as well as contribution types such as dictionary entries and announcements were removed from the corpus (-158). From the remaining publications, relevant contributions were selected on the basis of an inclusion criterion, which was checked against the respective abstract [22], [23]. The inclusion question was: Are barriers to the spread of digital solutions in the healthcare system or the basic parameters for digital innovations in the German healthcare system being investigated? (-191). In a final acquisition step, this selection was subjected to a backward reference search $(+3)$. One publication was not accessible $(-1)$, creating an overall literature corpus of 21 publications.

Inductive category formation is used to map the material on propagation barriers distortion-free [18]. For the first step of the category formation, the aim of the process has to be defined. The aim is to extract the barriers to propagation of DHIs in the German healthcare system from the body of literature, consisting of 21 publications. Next, a selection criterion will be defined to determine which passages of text are used to induce the formation of categories. If sections are found that can be assigned to the selection criterion, the first category is formulated as close as possible to the text formulation and confirm to the level of abstraction. Further categories are formed from the following text passages that fit into the selection if they can not be subsumed under existing categories. The categories are revised in an intermediate step before the material is completely worked through and the categorization can be interpreted. The result is depicted in Table. 1.

Analysis of existing assessment approaches. A second systematic literature search was carried out to record the state of research on evaluating the spread of digital health solutions on November 12, 2018 [17], [21]. Into this search, both, scientific contributions $(+225)$ and grey literature $(+20)$ were included. The 
Table.1 Categories of propagation barriers

\begin{tabular}{|l|l|}
\hline \multicolumn{2}{|c|}{ Super-categories } \\
$\begin{array}{l}\text { (1) Reimbursement and } \\
\text { Financing }\end{array}$ & $\begin{array}{l}\text { Remuneration conditions cross-sectoral; Remuneration conditions in the stationary sector; } \\
\text { Reimbursement in the outpatient sector; Non-remuneration of cost savings; Infrastructure } \\
\text { costs; High initial costs; Low willingness to pay on the second healthcare market [19]-[23] }\end{array}$ \\
\hline (2) Regulations and Guidelines & $\begin{array}{l}\text { Health market approval conditions; Legal data protection regulations; Lack of standardised } \\
\text { regulations: Liability risks; Ban on remote treatment [19]-[21], [24] }\end{array}$ \\
\hline (3) Technical Barriers & $\begin{array}{l}\text { Technical maintenance; Infrastructural barriers; Lack of security/reliability of medical data; } \\
\text { Lack of technical interoperability/compatibility [19]-[22] }\end{array}$ \\
\hline (4) Proof of Effectiveness & $\begin{array}{l}\text { Deficit in studies; Missing certification method; Lack of evidence of efficacy; Lack of } \\
\text { evaluation methodology [20]-[23] }\end{array}$ \\
\hline (5) Proof of Efficiency & Lack of efficiency evidence; Duration of efficiency assessment [23] \\
\hline (6) User Acceptance & $\begin{array}{l}\text { Knowledge and competence-related barriers; Insufficient relative advantage; Necessary } \\
\text { process changes; Resistance of the practitioner to changes in established practices; Questions } \\
\text { of trust towards the provider; Unsuitable organizational structure of the adopters; } \\
\text { Stigmatization of the patient; Reading/spelling deficit of the patient; Conservative attitude of } \\
\text { physicians towards innovations; Lack of technical affinity or knowledge among physicians } \\
\text { and patients; Fear of job loss on the part of the physician [19]-[22],[25]-[27] }\end{array}$ \\
\hline
\end{tabular}

search queries combined (1) types of digital health innovations (eHealth, telemedicine, etc.), (2) artifact types (framework, model, etc.) and (3) an element related to evaluation (quality management, evaluation, etc.). The search was carried out in the scientific databases PubMed, ScienceDirect, EBSCOhost and SpringerLink, as well as for grey literature with the search engine Google. Contributions were selected qualitatively for closer examination (-218). A forward reference search revealed additional relevant literature $(+4)$. The second literature corpus, consisting of 31 publications, was categorized using a taxonomy to get an overview of the scope of preliminary work at transparent standards [19].

Synthesis of the DHI assessment approach. Investigating existing evaluation approaches of digital health innovations, revealed that no distinct tool helping to address propagation barriers in the German healthcare system exists. This paper sets out to fill the lack of a Germany-centered solution to help practitioners dispatch propagation issues of their health innovations. Therefore, an evaluation sheet will be developed to assess how effectively barriers to the spread of digital health applications were dealt with. Since the draft to be designed contains an evaluation aspect, the Roadmap for Planning an Evaluation Concept for the Area of EHealth is used for the development of the artifact [24]. Complying with DSR-guidelines, the approach is finally demonstrated and evaluated [16].

\section{Status of research}

Research on the propagation barriers as well as evaluation approaches for DHIs already exist. This section presents an overview of these publications and derives barriers under consideration in this article from the literature base. Afterward, existing assessment frameworks are scrutinized according to their ability to suit the requirements imposed by the environment the German healthcare system operates in.

Identification of barriers to the spread of digital health innovations in Germany. Based on the first literature search, propagation barriers for innovations are identified through inductive category formation. Since the formed categories shall reflect propagation barriers, the selection criterion is: Propagation barrier for digital health innovations that are unique to the healthcare context and can be transferred to the German context. Categories that specifically refer to a non-German context are not formed (e.g. barriers from guidelines in sub-Saharan Africa). Thus, 33 categories were identified. In summary, six super-categories were formed (see also Table.1 for details):

(1) Reimbursement and Financing relates to barriers associated with monetary and budgeting issues as well as reimbursement of digital solutions in the public healthcare system [11], [25]-[28]. (2) Regulations and Guidelines sum up hurdles posed by statutory compulsions that have to be complied with [11], [25], [26], [29]. (3) Technical Barriers originate in technological restrictions or difficulties [11], [25]-[27], while (4) Medical Proof of Effectiveness confirms the medical properties of an innovation in healthcare [11], [26]-[28]. (5) Economic Proof of Efficiency encompasses issues around validating the return of investment of an innovation. [28] (6) User Acceptance focuses on social and organizational factors influencing the propagation of DHIs [11], [25]-[27], [30]-[32]. 
Mapping of existing DHI assessment approaches. Subsequently, a taxonomy was created to categorize the preliminary work containing evaluation approaches of DHIs. The aim is to find out to what extent the mentioned barriers to propagation have already been considered in evaluations of other authors. The taxonomy was created in seven iteration steps, resulting in seven taxonomy dimensions [19].

The first iteration distinguishes whether a contribution is applicable to a practical problem (Concrete), or whether it is a draft, a requirements analysis, a naming of critical factors, a recommendation or a plea for evaluation (Abstract). The second iteration differentiates between objects under consideration along the span of DHIs, as described in Otto et al. 2018. Iteration number three distinguishes between the intended geographical sphere of influence of the contribution (Global/No Specification, Developed NonEU Country, Developing Country, EU, Non-German $E U$-country and Germany). The next iteration creates a dimension to document the extent to which a contribution addresses the propagation barriers earlier identified with inductive category formation. The fifth iteration differentiates whether a contribution considers the overall quality of an intervention concept (Quality of the Intervention Concept), whether it supports an increased spread of the intervention (Diffusion Related), whether it focuses on the condition of an innovation (State Evaluation) or the successful implementation of the intervention (Successful Implementation). Iteration number six distinguishes whether an evaluation approach makes a summary statement (Summative), whether it is conducted in parallel to an implementation of the measure and influences it (Formative), or whether a contribution explores evaluation abstractly and no statement can be assigned to the mode (e.g. a plea for alignment of evaluation with certain standards, Too Abstract for a Mode) [18]. The last iteration categorizes contributions proposing or delivering a quantitative (Ordinal) or a qualitative (Nominal) evaluation result. A publication under consideration may be too vague to make a statement about the evaluation result (Too Abstract for a Result). The resulting taxonomy displaying the identified dimensions and characteristics is depicted in Table.2.

The taxonomy was applied to describe and categorize 31 identified assessment approaches in the second literature search (Section 2 Analysis of existing assessment approaches). The results reveal that so far, no contribution has been made that allows assessing which propagation barriers were being addressed by a DHI in the context of the German healthcare system. ${ }^{1}$

\footnotetext{
${ }^{1}$ The complete list of analyzed assessment approaches and results of applied taxonomy will be provided as an additional data source.
}

That implies that only little guidance exists for practitioners in this field to support development efforts and make a DHI fit for seamless spread. Therefore, an adequate evaluation approach is developed in the next section.

\section{Synthesizing the evaluation approach}

An evaluation approach helps stakeholders to assess the extent to which potential for scaling-up an innovation is being exploited and supports with recommending measures for successful market diffusion. This goal is worked towards in this section by synthesizing an evaluation approach that offers the opportunity to gather propagation barrier-related information about DHIs systematically and produce quantitative results indicating the innovation's barrier resilience [24]. The approach is designed in accordance with the evaluation standards Usefulness, Feasibility, Fairness and Accuracy [33].

For the artifact design, an existing scoring system will be altered and refined as intended in a DSR-artifact iteration [16]. This scoring system originally is the backbone of the MAPS-Tool (mHealth Assessment and Planning for Scale, WHO 2015), a framework examining mHealth-solutions focussing on their suitability for scaling-up along defined categories (Axis of Scale). Each category contains several globally applicable questions on the measures taken to achieve scaling goals. The quantification system that was employed is a four-stage point system.

Each category is rated depending on the response option picked by the surveyor: No (0 points), In Progress (1 point), Executed (2 points); Documented (3 points) or Not Applicable (3 points). Non-fulfillment $(\mathrm{No})$ expresses the previous disregard of the barrier aspect and is thus interpreted negatively. From there the rating increases leading to the documented confrontation with barriers (Documented), relating to testified efforts being made, and thus scores highest due to its traceability. If questions are not applicable, they are assessed positively to avoid influencing the overall picture unfavorably. Since five criteria are asked for each barrier, each of which is evaluated with up to three points, the evaluation sheet is based on a 15-point system. The modification of the MAPS-Tool concerns the frame of reference of the evaluation, which is alienated from generic global criteria and finds its unique feature compared to other approaches through the special reference to German propagation barriers. 
Table.2 Taxonomy of existing evaluation approaches for digital health innovations

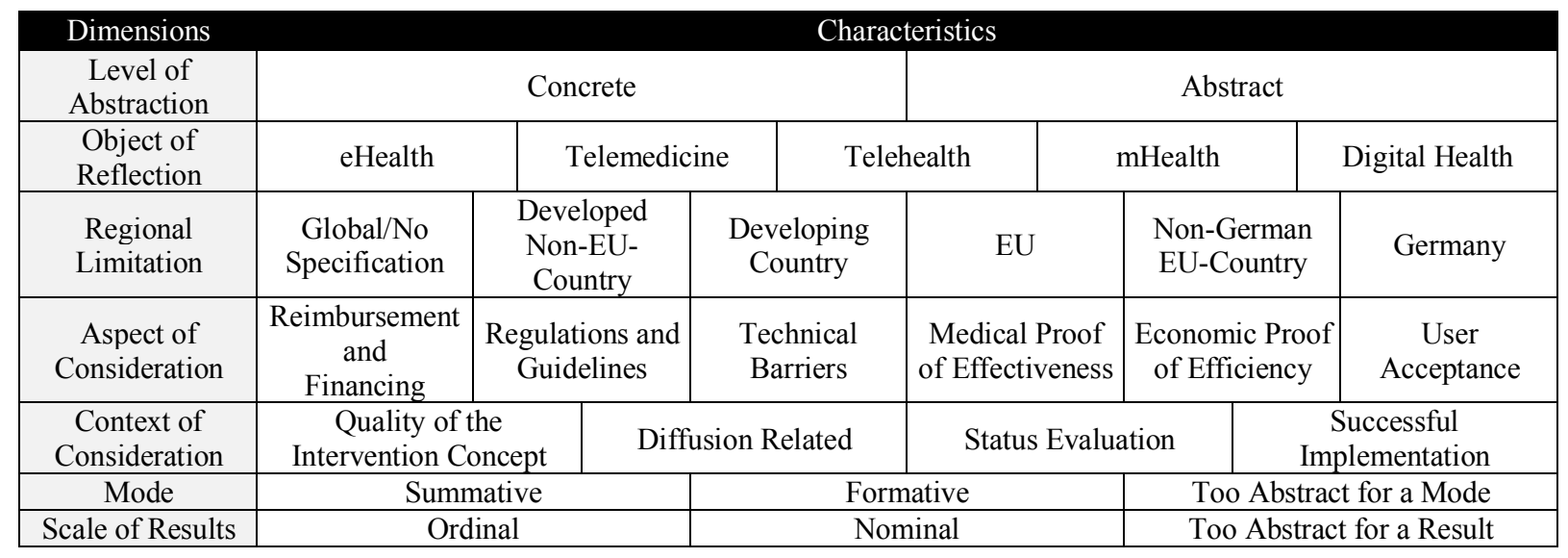

Furthermore, it is made applicable for every kind of DHI, including mHealth, telemedicine, telemonitoring, eHealth and alike [10]. Thus, a thorough analysis of the German healthcare system was conducted along the barriers for DHIs to concretize the six super-categories in appropriate assessment items. The adaptation of the following assessment approach to other national specifications requires an appropriate adjustment of this step of analysis and design.

(1) Reimbursement and Financing was covered with an analysis of possibilities for reimbursement in the ambulant and stationery sector as well as the intersectoral reimbursement.

(2) Regulations and Guidelines incorporate findings related to the E-Health-Law (SGB V), like the registration of DHIs in the a public online interoperability register (gematik's vesta information portal [34]), as well as data security regulations (DSGVO/GDPR), laws regarding medical products including Conformité Européenne (CE) certification, and IT security regulations with policies for critical infrastructure (KRITIS).

(3) Technical Barriers were considered including technical (HL7, DICOM, xDT) and semantic communication standards (OPS, ICD, SNOMED CT, LOINC) as well as technical security requirements for medical devices (93/42/EWG) and data security considerations ( $§ 64$ BDSG). Additionally, transmission technology and special user needs have to be considered.

(4) Proof of Medical Effectiveness contains measures assuring compliance with the principle of evidence-based medicine in the public healthcare system, the role of clinical trials $(93 / 42 / \mathrm{EWG})$ and peculiarities of health technology assessments (HTAs).

\footnotetext{
${ }^{2}$ Information about mySugr has been collected from company websites. Details may differ due to national specifications.
}

(5) Economic Proof of Efficiency encompasses actions towards the efficiency command ( $\$ 12$ SGB V) in the German public healthcare system and variations of health-economic analysis as well as cost types to prove equal or better care provision for lower costs.

Finally, (6) User Acceptance utilized the Technology Acceptance Model to analyze patient and practitioner needs [35]. The resulting assessment form can be seen in Table.3. The scores for the aspects in question can be documented in the left column.

\section{Demonstration}

The newly developed artifact will be demonstrated on a phenotypical digital health solution with a user base in Germany. The case study is based on the application mySugr: your intelligent diabetes diary. Among the functions of $\mathrm{mySugr}^{2}$ are collecting and graphically processing information on diabetes (e.g. blood sugar, meals, medication, physical activities) in the form of a diary to optimize therapy outcomes and mitigate the course of the disease [36].

1) Reimbursement and Financing. mySugr is a medical device and therefore belongs to the first health market [37]. The app is available in a free basic version and can be extended in its functions by using a version with costs. Since April 2018, some private health insurance companies reimbursed the costs for using the application [38]; from July 2018, this was also offered by a selection of public health insurance companies. Various financing models of the public and private sectors were considered, implemented and documented. Patient-financed, as well as health insurance-financed models, are available. Score: 15 
Table.3 Assessment form for DHI-applications

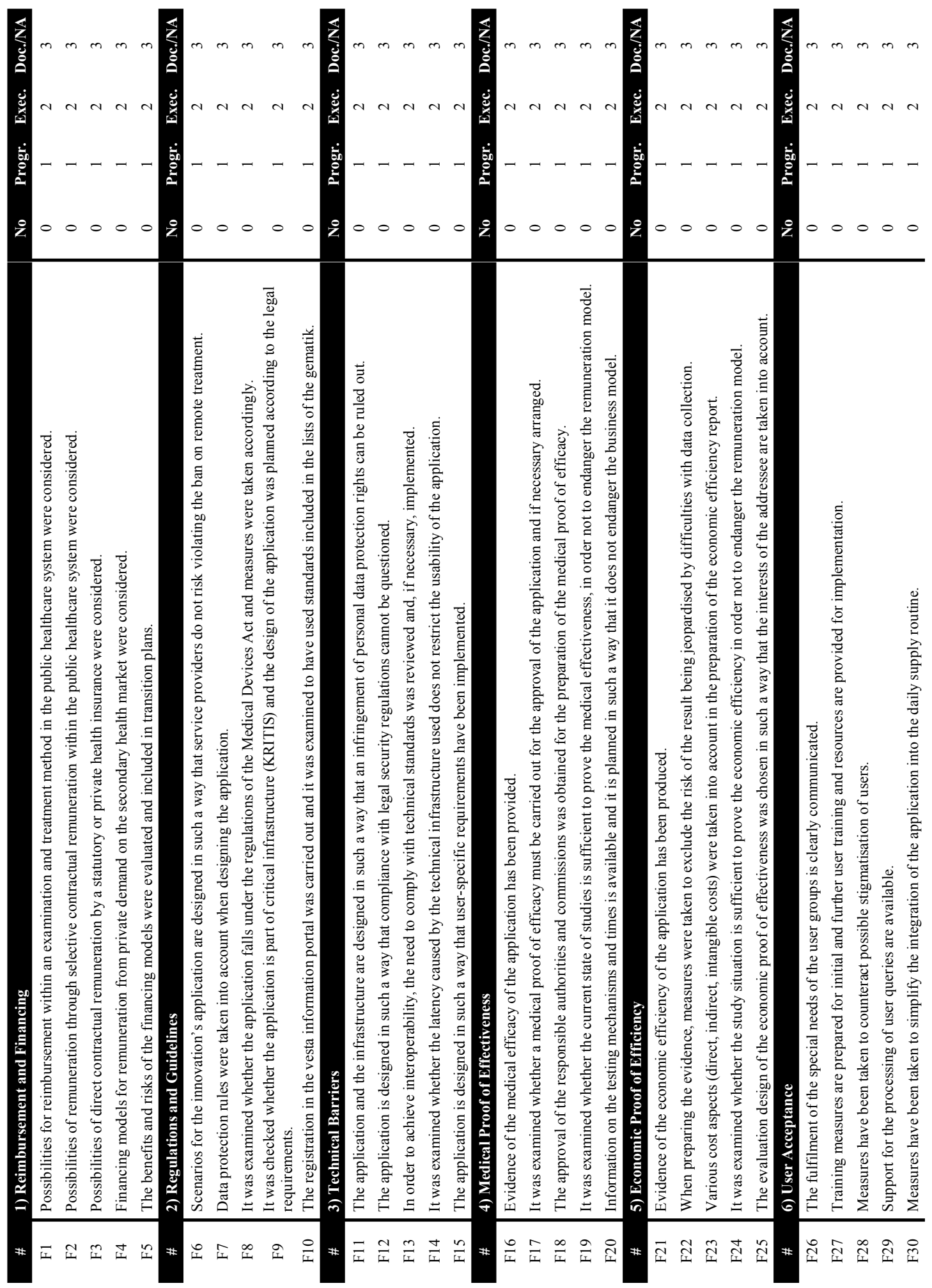


2) Regulations and Guidelines. The application is designed as a tracker and diary of diabetes data and does not include remote treatment functions [39]. mySugr ensures compliance with various data protection guidelines in the data protection declaration [40]. In addition, the app is approved as a Class I medical device (one module as Class II). The application is not critical infrastructure so that special requirements for KRITIS do not apply. Deductions apply because mySugr is not registered in the vesta Informationsportal. Score: 12

3) Technical Barriers. The technical design of the diabetes tracker for the protection of personal data is reassured in company statements [41], with reference to the use of the Amazon Web Services cloud infrastructure and its certificates and compliance with standards and guidelines. The security design is again proven by the $C E$ conformity of mySugr. The application is interoperable with various blood glucose monitoring devices [39]. The transmission of larger amounts of data does not result from the application's use cases so that latency times do not have to be considered. The user ratings confirm that the app mostly fulfills user-specific requirements [43]. Number of points: 13

4) Medical Proof of Efficacy. The medical efficacy of mySugr has been demonstrated and published [44], [45]. Thereby, the present state of studies was improved, and the reimbursement model was backed-up with clinical data. Testing times and mechanisms did not pose a lasting threat to the existence of the project. Score: 15

5) Economic Proof of Efficiency. Support from investors for the development team indicates proved efficiency. Financing rounds between 2014 and 2015 ended with a monetary backing of several million euros [46] and culminated in the takeover by Roche in 2017 [47]. Score: 15

6) User Acceptance. The application is specifically designed to meet the documentation needs of diabetics. Despite the very good ratings on Google Play, there are hints from users in the comments about the app's inadequacies [43]. The mySugr website offers training courses on how to use the app [48]. Stigmatization of users is prevented with a humorous character of application and the creation of a sense of community in the user base through blog entries and newsletters. Support is available on a variety of channels [49]. Studies were conducted to measure user satisfaction, in which the application was rated positively [50]. Score: 13

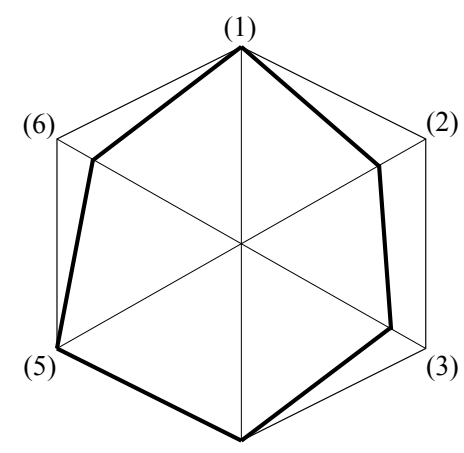

(4)

Figure 1. Visualization scoring "mySugr"

The demonstration showed that the presented approach to propagation barrier assessment is applicable to a popular DHI that is used on the German market. It helps to analyze which hurdles imposed on DHIs by the German healthcare system were addressed by mySugrdevelopers and which would still need some attention in order to speed up the spread of the application and reach a higher volume of users.

\section{Discussion}

Based on the results of the former sections, the designed assessment approach is evaluated with respect to the standards of the German association for evaluation [33]. Usefulness. The result of the assessment of mySugr coincides with the actual successful propagation development of the application. The next step would be to examine low scored areas and take measures to address under-considered barriers. Whether those measures lead to practice-relevant changes for the application could not be clarified in the demonstration. However, the evaluation remains a visible confirmation for outsiders reflecting the strong positioning of mySugr, which presents a benefit. Feasibility. The feasibility of the assessment largely depends on the robustness of the information base for the solution under consideration. In the case of mySugr, the company's tendency to openly communicate with its userbase contributed to a highly feasible assessment. However, with the knowledge of a company insider, statements could have been made more accurately. Fairness. Measures must be taken to ensure that all information necessary for an assessment is available to all stakeholders at all times, i.e. that assessments of solutions take place under the same (fair) conditions for third parties. One possibility could be to access standardized data for assessment in the vesta Informationsportal, although it is often provided insufficiently. In order to preserve the feasibility of an evaluation to cover the full range of DHIs, fairly generic evaluation questions had to be used which could have 
compromised the accuracy of the evaluation of an mHealth application. Accuracy. The assessment of mySugr resulted in an overall positive score indicating that most of the propagation barriers were addressed and dealt with, which is reflected in the successful market dissemination of the app. However, compromises had to be made regarding the assessment parameters (No, In Progress, Executed, Documented/Not Applicable). They are not linked to the quality of a measure taken and its sustainable effectiveness, which affects the effective barrier resilience of an application. Therefore, more resilient metrics for the assessment of barrier aspects have to be introduced.

This article contributes to diffusion and adoption research in healthcare. It offers insides into the aspects of healthcare-specific diffusion barriers by integrating former research in the area and applying a distinct focus on the German environment. The resulting categorization offered a frame for investigating the German healthcare system under more narrow thematic limitations. Additionally, a review of preliminary research on healthcare innovation barriers was provided. These two preparatory steps lead to the design of an easy-to-use approach to assess the readiness of an innovation to successfully spread. Usefulness and functionality of DHI play only a secondary role in this approach. Sustainable value is rather added by shifting from functionality-focused thinking towards dissemination-centered considerations. Summarizing this paper's scientific relevance: it enriches existent knowledge about DHI diffusion into healthcare markets by a formative assessment method that allows the determination of how a DHI may take the hurdles of concrete diffusion barriers. In a broader sense, the presented measures imply design principles of DHI.

The presented artifact is aligned with the $\mathrm{EU}$ interoperability framework. The proposed evaluation approach supports innovators and development teams struggling with the complexity of DHI diffusion into day-to-day healthcare. As interoperability is considered highly relevant to ensure DHI diffusion success, research and consensus processes of eHealth Network (a task network of the European Commission) led to the Refined European Interoperability Framework (ReEIF) in 2015 as a general definition for eHealth interoperability. The contribution of this paper is aligned with this consensus as categories and supercategories address the six interoperability levels of ReEIF (Legal and regulatory; Policy; Care Process; Information; Applications; IT-Infrastructure) [51]. Thus, the proposed assessment approach breaks down the complexity of DHI diffusion while offering an aid kit for struggling innovators.
The presented artifact is also subject to limitations. One limitation results from the methodology of the literature-based research approach deriving barriers with inductive category formation. This practice results in categories formed on the one hand regardless of contemporary significance, on the other hand relying on the assumption that relevant barriers were already being discovered in preliminary research. Thus, it can not be guaranteed that the barriers found have practical relevance, which requires research in the field and can be worked on by conducting expert interviews. Additionally, the categories that were formed and represent propagation barriers exist in de facto interdependence but were considered as being separate in this paper. That could lead to false compartmentalization of the healthcare system during the attempt to create the assessment approach. Another basic potentially misleading assumption was created around the unified understanding of the terminology summed up in the term digital health during the research on existing evaluation frameworks. Thus, subsequent categorizations of named approaches might have suffered from ambiguity. Furthermore, the evaluation revealed that the quality of the information fed into the assessment model is pivotal for the assessment outcome. Hence the reliability of the information used in the artifact application has to be ensured. Moreover, proof is required that the assessment approach followed by the scoring system does indeed support hands-on decision making. This is especially true, since the descriptions of the healthcare environment the assessment is based on, was only briefly broached and is currently not to the innovator's disposal in case a category scored low. Finally, the frame in which this approach to DHI assessment is tailored to is currently limited to the German healthcare context. That limitation was necessary to create a usefully specific, manageable framework in the first place, seeing the German healthcare system as fairly homogeneous. Since that focus was set in such an early stage of research, the migration of the assessment framework to another region might require fundamental content changes early on. However, the methodology would be just as applicable and an expansion to other OECD member states with Social Health Insurance [15] might require slight modifications only.

\section{Conclusion}

This paper discussed a central issue of digital health innovations - even highly optimized DHIs with strong problem-solving potential do not necessarily scale-up in the intended environment. A crucial factor for that are system-imposed barriers that have to be taken into consideration to introduce innovations to end-users and 
unfold expected benefits for care provision. To tackle this issue, a first draft of an evaluation approach was developed to determine the resilience of DHIs to propagation barriers in the German healthcare system. Apart from improving the approach by additional research on the limitations, the assessment can be extended to a stakeholder perspective by linking propagation barriers to corresponding interest groups in the healthcare system. These interest groups often have contradicting goals and different levels of influence on DHI-diffusion success. Thus, the extension would benefit the overall approach by weighting barriers stakeholder-sensitively which adds more accuracy to the scoring. In practice, the presented approach could be implemented as an evaluation tool on a platform for digital health solutions using the already available ordinal scoring or adding a nominal dimension, describing addressable barrier aspects based on the assessment in greater detail. Such services could either provide guidance to developers working on DHIs or assist with investment decisions into promising innovations. Thus, the approach introduced in this paper can contribute to using scarce resources in healthcare more sustainably.

\section{Acknowledgment}

This work is part of the EFRE-funded project „Häusliche Gesundheitsstation” supported by the European Union and Free State of Saxony. The authors thank the project partners for the good cooperation.

\section{References}

[1] United Nations, "The Millennium Development Goals Report," United Nations, p. 72, 2015, doi: 978-92-1101320-7.

[2] Y. M. Asi and C. Williams, "The role of digital health in making progress toward Sustainable Development Goal (SDG) 3 in conflict-affected populations." International Journal of Medical Informatics, 2017, [Online]. Available:

http://dx.doi.org/10.1016/j.ijmedinf.2017.11.003.

[3] WHO, WHO guideline: recommendations on digital interventions for health system strengthening. Geneva, 2019.

[4] Federal Ministry of Health, "Digital Healthcare Act (DVG)," Digital Healthcare Act (DVG): Driving the digital transformation of Germany's healthcare system for the good of patients, Dec. 03, 2019. https://www.bundesgesundheitsministerium.de/digitalhealthcare-act.html.

[5] L. Otto and L. Harst, "Investigating Barriers for the Implementation of Telemedicine Initiatives: A Systematic Review of Reviews," p. 10, 2019.

[6] D. Horgan, H. J. van Kranen, and S. A. Morré, "Optimising SME Potential in Modern Healthcare
Systems: Challenges, Opportunities and Policy Recommendations," Public Health Genomics, vol. 21, no. 1-2, pp. 1-17, 2018, doi: 10.1159/000492809.

[7] Bellagio eHealth Evaluation Group, "WHO Global eHealth Evaluation meeting, organized in Bellagio, Italy, September 7-9, 2011." eHealth Evaluation Evidence, 2011.

[8] E. M. Rogers, Diffusion of Innovations, 5th Edition. Free Press, 2003.

[9] P. Uvin, "Fighting hunger at the grassroots: Paths to scaling up," World Development, vol. 23, no. 6, pp. 927939, 1995, doi: 10.1016/0305-750X(95)00028-B.

[10] L. Otto, L. Harst, H. Schlieter, B. Wollschlaeger, P. Richter, and P. Timpel, "Towards a Unified Understanding of eHealth and Related Terms - Proposal of a Consolidated Terminological Basis," Proceedings of the 11th International Joint Conference on Biomedical Engineering Systems and Technologies, vol. 2, no. 2011, pp. 533-539, 2018, doi: 10.5220/0006651005330539.

[11] T. H. Broens, R. M. Huis in't Veld, M. M. VollenbroekHutten, H. J. Hermens, A. T. van Halteren, and L. J. Nieuwenhuis, "Determinants of successful telemedicine implementations: a literature study," Journal of telemedicine and telecare, vol. 13, no. 6, pp. 303-309, 2007.

[12] R. Nouri, S. R Niakan Kalhori, M. Ghazisaeedi, G. Marchand, and M. Yasini, "Criteria for assessing the quality of mHealth apps: a systematic review," J Am Med Inform Assoc, vol. 25, no. 8, pp. 1089-1098, Aug. 2018, doi: 10.1093/jamia/ocy050.

[13] T. Kowatsch, L. Otto, S. Harperink, A. Cotti, and H. Schlieter, "A design and evaluation framework for digital health interventions," it - Information Technology, vol. 0, no. 0, Nov. 2019, doi: 10.1515/itit-2019-0019.

[14] K. Peffers et al., "The design science research process: A model for producing and presenting information systems research," in Proceedings of the First International Conference on Design Science Research in Information Systems and Technology (DESRIST 2006), Claremont, CA, USA, 2006, pp. 83-106.

[15] K. Böhm, A. Schmid, R. Götze, C. Landwehr, and H. Rothgang, "Five types of OECD healthcare systems: Empirical results of a deductive classification," Health Policy, vol. 113, no. 3, pp. 258-269, Dec. 2013, doi: 10.1016/j.healthpol.2013.09.003.

[16] Hevner, March, Park, and Ram, "Design Science in Information Systems Research," MIS Quarterly, vol. 28, no. 1 , p. 75 , 2004, doi: $10.2307 / 25148625$.

[17] J. vom Brocke, A. Simons, B. Niehaves, B. Niehaves, and K. Reimer, "Reconstructing the Giant: On the Importance of Rigour in Documenting the Literature Search Process," 2009, [Online]. Available: http://aisel.aisnet.org/ecis2009\%0Ahttp://aisel.aisnet.org /ecis2009/161.

[18] P. Mayring, "Qualitative content analysis: theoretical foundation, basic procedures and software solution," 2014.

[19] R. C. Nickerson, U. Varshney, and J. Muntermann, “A method for taxonomy development and its application in information systems," no. April 2012, pp. 336-359, 2013, doi: 10.1057/ejis.2012.26. 
[20] WHO, "The MAPS Toolkit: mHealth Assessment and Planning for Scale," p. 106, 2015.

[21] H. M. Cooper, "Organizing knowledge synthesis: a taxonomy of literature reviews," Knowledge in society, vol. 1, no. 1, pp. 104-126, 1988, doi: 10.1007/BF03177550.

[22] B. Kitchenham, "Procedures for performing systematic reviews," Keele, UK, Keele University, vol. 33, no. TR/SE-0401, p. 28, 2004, doi: 10.1.1.122.3308.

[23] R. J. B. Vanwersch et al., "Methodological support for business process redesign in health care: a literature review protocol," International Journal of Care Pathways, vol. 15, no. 4, pp. 119-126, 2011, doi: 10.1258/jicp.2011.011025.

[24] J. Köberlein-Neu and S. Müller-Mielitz, "Roadmap zur Entwicklung eines Evaluationskonzeptes," S. MüllerMielitz and T. Lux, Eds. Berlin Heidelberg New York: Springer-Verlag, 2017, pp. 881-892.

[25] J. G. Anderson, "Social, ethical and legal barriers to Ehealth," International Journal of Medical Informatics, vol. 76, no. 5-6, pp. 480-483, 2007, doi: 10.1016/j.ijmedinf.2006.09.016.

[26] S. Becker, T. Miron-Shatz, N. Schumacher, J. Krocza, C. Diamantidis, and U.-V. Albrecht, "mHealth 2.0: Experiences, Possibilities, and Perspectives," JMIR mHealth and uHealth, vol. 2, no. 2, pp. 1-12, 2014, doi: 10.2196/mhealth.3328.

[27] P. J. Batterham et al., "Developing a roadmap for the translation of e-mental health services for depression.," Australian \& New Zealand Journal of Psychiatry, vol. 49, no. 9, pp. 776-784, Sep. 2015.

[28] A. M. Fraiche, Z. J. Eapen, and M. B. McClellan, "Moving Beyond the Walls of the Clinic: Opportunities and Challenges to the Future of Telehealth in Heart Failure," JACC: Heart Failure, vol. 5, no. 4, pp. 297-304, 2017, doi: 10.1016/j.jchf.2016.11.013.

[29] V. Rowthorn and D. Hoffmann, "Legal impedements to the diffusion of telemedicine," Journal of Health Care Law \& Policy, vol. 14, pp. 1-53, 2011.

[30] J. Walker and S. Whetton, "The diffusion of innovation: factors influencing the uptake of telehealth," vol. 8, pp. 75-77, 2002.

[31] H. Tanriverdi and C. S. Iacono, "Knowledge Barriers To Diffusion of Telemedicine," the international conference on Information systems, pp. 39-50, 1998.

[32] M. Kifle, V. W. A. Mbarika, and R. V. Bradley, "Global Diffusion of the Internet $\mathrm{X}$ : The Diffusion of Telemedicine in Ethiopia: Potential Benefits, Present Challenges, and Potential Factors," Communications of the Association for Information Systems, vol. 18, no. 1, pp. 612-640, 2006.

[33] DeGEval, Standards für Evaluation, 1st ed. Mainz: Druckerei Zeidler, 2016.

[34] Gematik, "Entgeltkatalog für die Aufnahme von Informationen in das Interoperabilitätsverzeichnis," pp. $1-5,2018$.

[35] F. D. Davis, "A Technology Acceptance Model for empirically testing new end-user information systems: theory and results," 1985, doi: oclc/56932490.

[36] mySugr, "3 Gründe warum man ein Diabetes-Tagebuch führen sollte," 2019. https://mysugr.com/de-de/blog/3- grunde-warum-man-ein-diabetes-tagebuch-fuhren-sollte (accessed Nov. 28, 2019).

[37] mySugr, "Diabetes sollte Nebensache sein, automatisch nebenher laufen," 2019. https://mysugr.com/dede/blog/diabetes-sollte-nebensache-sein-automatischnebenher-laufen (accessed Nov. 28, 2019).

[38] Ärzte Zeitung online, "Private Kassen übernehmen Kosten für Diabetes-App," 2018, Accessed: Feb. 10, $2019 . \quad$ [Online]. Available: https://www.aerztezeitung.de/politik_gesellschaft/krank enkassen/article/961058/medizinprodukte-privatekassen-uebernehmen-kosten-diabetes-app.html.

[39] mySugr, "Pro," 2019, Accessed: Feb. 10, 2019. [Online]. Available: https://mysugr.com/de/apps/pro/.

[40] mySugr, "Datenschutzerklärung," 2018, Accessed: Feb. 10, 2019. [Online]. Available: https://legal.mysugr.com/documents/privacy_policy_eu/ current.html?.ga=2.75877725.91677720.1537362238669256811.1524663864 .

[41] mySugr, "Was passiert bei mySugr eigentlich mit meinen Daten?" https://mysugr.com/de-de/blog/datenschutzsicherheit (accessed Nov. 27, 2019).

[42] mySugr, "FAQ Datenimport," 2019, Accessed: Feb. 10, $2019 . \quad$ [Online]. Available: https://support.mysugr.com/hc/de/categories/202576347 -Datenimport.

[43] Google, "mySugr - Diabetes App \& Blutzucker Tagebuch - Apps bei Google Play,” 2019. https://play.google.com/store/apps/details?id=com.mysu gr.android.companion\&hl=de (accessed Nov. 27, 2019).

[44] M. Hompesch, G. Scheiner, L. Schuster, J. Kober, and F. Debong, "Clinically-Relevant Improvement in Quality of Blood Glucose Control in Well-Controlled Users of mySugr's Mobile Diabetes Management Tool," 2018, doi: $10.2337 / \mathrm{dc} 08-0878$.

[45] M. Hompesch, K. Kalcher, F. Debong, and L. Morrow, "Significant Improvement of Blood Glucose Control in a High Risk Population of Type 1 Diabetes Using a Mobile Health App - A Retrospective Observational Study," 2017, doi: $10.2337 / \mathrm{dc} 08-0878.4$.

[46] mySugr, "The Viennese startup snaps up over Euro 1 million in investment," Wien, 2014.

[47] mySugr, "mySugr joins the Roche Family," 2017, Accessed: Feb. 10, 2019. [Online]. Available: https://mysugr.com/hello-roche/.

[48] mySugr, "mySugr Tagebuch - ein kleiner Rundgang durch die App," 2015, Accessed: Feb. 10, 2019. [Online]. Available: https://mysugr.com/de/durch-loggen-demmonster-die-stirn-bieten/.

[49] mySugr, "Support," 2019, Accessed: Feb. 10, 2019. [Online]. Available: https://support.mysugr.com/hc/de.

[50] J. Hibbits, J. Kober, J. Wrede, J. Belik, and F. Debong, "Strong Customer Satisfaction Among Users of Mobile Diabetes Management," 2018.

[51] eHealth Network, "Refined eHealth European Interoperability Framework," Eurupean Commission, Brussels, Nov. 2015. [Online]. Available: https://ec.europa.eu/health/sites/health/files/ehealth/docs lev_20151123_co03_en.pdf. 